\title{
ON THE ROOT FUNCTIONS OF GENERAL ELLIPTIC BOUNDARY VALUE PROBLEMS
}

\author{
N. TARKHANOV
}

\begin{abstract}
We consider a boundary value problem for an elliptic differential operator of order $2 m$ in a domain $\mathcal{D} \subset \mathbb{R}^{n}$. The boundary of $\mathcal{D}$ is smooth outside a finite number of conical points, and the Lopatinskii condition is fulfilled on the smooth part of $\partial \mathcal{D}$. The corresponding spaces are weighted Sobolev spaces $H^{s, \gamma}(\mathcal{D})$, and this allows one to define ellipticity of weight $\gamma$ for the problem. The resolvent of the problem is assumed to possess rays of minimal growth. The main result says that if there are rays of minimal growth with angles between neighbouring rays not exceeding $\pi(\gamma+2 m) / n$, then the root functions of the problem are complete in $L^{2}(\mathcal{D})$. In the case of second order elliptic equations the results remain true for all domains with Lipschitz boundary.
\end{abstract}

\section{INTRODUCTION}

Expansions over eigenvectors of selfadjoint operators in a Hilbert space are well understood. After [Mar62] expansions over root vectors are also intensively elaborated for weak perturbations of selfadjoint operators, cf. [Agr94a]. Although such investigations are of independent interest in operator theory, most interesting applications are still in elliptic theory, cf. [Agr94].

The problem of completeness of the system of eigen and associated functions of boundary value problems for elliptic operators in domains with smooth boundaries was studied in many articles.

The study was initiated by the paper of Keldysh [Kel51] who proved a general theorem on the completeness of the system of eigen and associated functions of non-selfadjoint differential operators and obtained as its corollary the theorem on the completeness for elliptic operators of second order with the Dirichlet boundary conditions.

In a series of papers [Bro53], [Bro59a], [Bro53b], Browder obtained the theorem for the Dirichlet problem for elliptic operators of arbitrary order with real principal part.

Agmon [Agm62] and Schechter [Sch59] proved that the system of root functions of an elliptic boundary problem is complete in a bounded domain $\mathcal{D}$ with a smooth boundary provided that the Lopatinskii condition is fulfilled. Recently Agranovich et al. [ADF00] improved the Agmon theorem by relaxing the regularity conditions on the boundary.

For the Dirichlet problem for strongly elliptic differential operators of order $2 m$ the completeness of the system of eigen and associated functions in $L^{2}(\mathcal{D})$, where $\mathcal{D}$ is an arbitrary bounded domain, was proved by Agranovich [Agr94a]. He studied also the problem with the Neumann boundary conditions, assuming that the surface $\partial \mathcal{D}$ is Lipschitzian.

In the case of Neumann problem for second order elliptic systems the completeness of root functions was investigated by Krukovsky [Kru76].

Date: July 12, 2005.

2000 Mathematics Subject Classification. Primary 35P10; Secondary 47A75.

Key words and phrases. Elliptic operators, Lipschitz domains, completeness of eigenfunctions. 
All the mentioned authors actually used the methods of [Kel51]. We shall also use them here as well as the methods of Carleman presented in [Car36].

\section{EXPANSION OF THE RESOLVENT}

Let $B$ be a Banach space and $\mathcal{L}(B)$ the algebra of all bounded linear operators acting in $B$.

Suppose $\lambda_{0} \in \mathbb{C}$ and $F(\lambda)$ is a holomorphic function in a punctured neighbourhood of $\lambda_{0}$ which takes its values in $\mathcal{L}(B)$.

The point $\lambda_{0}$ is called a characteristic value of $F(\lambda)$ if there exists a holomorphic function $u(\lambda)$ in a neighbourhood of $\lambda_{0}$ with values in $B$, such that $u\left(\lambda_{0}\right) \neq 0$ but $F(\lambda) u(\lambda)$ extends to a holomorphic function near $\lambda_{0}$ and vanishes at this point. We call $u(\lambda)$ a root function of $F(\lambda)$ at $\lambda_{0}$.

Assume that $\lambda_{0}$ is a characteristic value of $F(\lambda)$ and $u(\lambda)$ a root function at $\lambda_{0}$. The order of $\lambda_{0}$ as a zero of $F(\lambda) u(\lambda)$ is called the multiplicity of $u(\lambda)$, and the vector $u_{0}=u\left(\lambda_{0}\right)$ an eigenvector of $F(\lambda)$ at $\lambda_{0}$. If supplemented by the zero vector, the eigenvectors of $F(\lambda)$ at $\lambda_{0}$ form a vector space. The closure of the set of all eigenvectors of $F(\lambda)$ at $\lambda_{0}$ is called the kernel of $F(\lambda)$ at $\lambda_{0}$, and it is denoted by ker $F\left(\lambda_{0}\right)$.

By the rank of an eigenvector $u_{0} \in B$ is meant the maximum of the multiplicities of all root functions $u(\lambda)$ such that $u\left(\lambda_{0}\right)=u_{0}$, if the set of multiplicities of these functions is bounded. If these multiplicities are unbounded, the rank of $u_{0}$ is taken to be infinity.

Suppose that ker $F\left(\lambda_{0}\right)$ is of finite dimension $I$ and that the ranks of all eigenvectors $u_{0} \in \operatorname{ker} F\left(\lambda_{0}\right)$ are finite. By a canonical system of eigenvectors of $F(\lambda)$ at $\lambda_{0}$ we mean any system of eigenvectors $u_{0,1}, \ldots, u_{0, I}$ with the property that the rank of $u_{0,1}$ is maximal among the ranks of all eigenvectors of $F(\lambda)$ at $\lambda_{0}$ and the rank of $u_{0, i}$ is maximal among the ranks of all eigenvectors of $F(\lambda)$ at $\lambda_{0}$ in any direct complement in ker $F\left(\lambda_{0}\right)$ of the linear span of the vectors $u_{0,1}, \ldots, u_{0, i-1}$, for $i=2, \ldots, I$.

Let $r_{i}$ be the rank of $u_{0, i}$. It is easy to see that the rank of any eigenvector $u_{0}$ corresponding to the characteristic value $\lambda_{0}$ is equal to one of the $r_{i}$. Consequently, the numbers $r_{1}, \ldots, r_{I}$ are uniquely determined by the function $F(\lambda)$. Note that a canonical system of eigenvectors is not uniquely determined in general. The numbers $r_{i}$ are said to be partial null multiplicities of the characteristic value $\lambda_{0}$ of $F(\lambda)$. Following [GS71], we call $n\left(F\left(\lambda_{0}\right)\right)=r_{1}+\ldots+r_{I}$ the null multiplicity of the characteristic value $\lambda_{0}$ of $F(\lambda)$. If $F(\lambda)$ has no root functions at $\lambda_{0}$, we set $n\left(F\left(\lambda_{0}\right)\right)=0$.

We now apply these arguments again, with $F(\lambda)$ replaced by the inverse family $F^{-1}(\lambda)$. Suppose $\lambda_{0} \in \mathbb{C}$ is a characteristic value of $F^{-1}(\lambda)$ and the kernel of $F^{-1}(\lambda)$ at $\lambda_{0}$ is of finite dimension $J$. If $\varrho_{1}, \ldots, \varrho_{J}$ are the partial null multiplicities of this characteristic value of $F^{-1}(\lambda)$, then we call $\varrho_{1}, \ldots, \varrho_{J}$ the partial polar multiplicities of the characteristic value $\lambda_{0}$ of $F(\lambda)$. We call $n\left(F^{-1}\left(\lambda_{0}\right)\right)=\varrho_{1}+\ldots+\varrho_{J}$ the polar multiplicity of the characteristic value $\lambda_{0}$ of $F(\lambda)$ and denote it by $p\left(F\left(\lambda_{0}\right)\right)$. If $F^{-1}(\lambda)$ has no root functions at $\lambda_{0}$, we set $p\left(F\left(\lambda_{0}\right)\right)=0$.

The quantity $m\left(F\left(\lambda_{0}\right)\right)=n\left(F\left(\lambda_{0}\right)\right)-p\left(F\left(\lambda_{0}\right)\right)$ is called the multiplicity of the characteristic value $\lambda_{0}$ of $F(\lambda)$.

If $F(\lambda)$ is holomorphic at the point $\lambda_{0}$ and the operator $F\left(\lambda_{0}\right)$ is invertible, then $\lambda_{0}$ is called a regular point of $F(\lambda)$. Note that the multiplicity of any regular point of $F(\lambda)$ is equal to zero.

In the scalar case it is evident that the multiplicity of a characteristic value $\lambda_{0}$ of a function $F(\lambda)$ is equal to the multiplicity of the zero if $\lambda_{0}$ is a zero of $F(\lambda)$, and is equal to the order of the pole if $\lambda_{0}$ is a pole. 
Assume that $\lambda_{0}$ is a pole of the operator-valued function $F(\lambda)$. In some neighbourhood of $\lambda_{0}$ we get an expansion

$$
F(\lambda)=\sum_{j=-m}^{\infty} F_{j}\left(\lambda-\lambda_{0}\right)^{j}
$$

where $F_{j} \in \mathcal{L}(B)$.

If in (1.1) the operators $F_{-1}, \ldots, F_{-m}$ are of finite rank, then $F(\lambda)$ is called finitely meromorphic at $\lambda_{0}$.

The operator-valued function $F(\lambda)$ is said to be of Fredholm type at the point $\lambda_{0}$ if the operator $F_{0}$ in the expansion (1.1) is Fredholm. This is equivalent to saying that the value of $F$ at $\lambda_{0}$ is a Fredholm operator.

A point $\lambda_{0}$ is called a normal point of $F(\lambda)$ if $F(\lambda)$ is finitely meromorphic and of Fredholm type at $\lambda_{0}$ and if all points of some punctured neighbourhood of $\lambda_{0}$ are regular for $F(\lambda)$.

By [GS71], each normal point $\lambda_{0}$ of $F(\lambda)$ is a normal point of $F^{-1}(\lambda)$. If, in addition, $\lambda_{0}$ is a pole of either $F(\lambda)$ or $F^{-1}(\lambda)$, then it is a characteristic value of finite multiplicity of the other.

Expanding $F(\lambda)$ and $u(\lambda)$ as Laurent series (1.1) and

$$
u(\lambda)=\sum_{k=0}^{\infty} u_{k}\left(\lambda-\lambda_{0}\right)^{k},
$$

respectively, we get

$$
F(\lambda) u(\lambda)=\sum_{n=-m}^{r-1}\left(\sum_{j+k=n} F_{j} u_{k}\right)\left(\lambda-\lambda_{0}\right)^{n}+O\left(\left|\lambda-\lambda_{0}\right|^{r}\right)
$$

close to $\lambda_{0}$. It follows that for $u(z)$ to be a root function of $F(\lambda)$ at $\lambda_{0}$ of multiplicity $r \geq 1$ it is necessary and sufficient that

$$
\sum_{k=0}^{n+m} F_{n-k} u_{k}=0
$$

for all $n=-m, \ldots, r-1$.

The derivatives

$$
u_{k}=\frac{1}{k !} u^{(k)}\left(\lambda_{0}\right)
$$

$k=1, \ldots, r-1$, are said to be associated vectors for the eigenvector $u_{0}=u\left(\lambda_{0}\right)$ of $F(\lambda)$ at $\lambda_{0}$. Any subsystem $u_{0}, u_{1}, \ldots, u_{s}$ with $s \leq r-1$ is called a Jordan chain of length $s+1$ of $F(\lambda)$ at $\lambda=\lambda_{0}$.

Suppose $u_{0,1}, \ldots, u_{0, I}$ is a canonical system of eigenvectors of $F(\lambda)$ at $\lambda_{0}, I$ being the dimension of ker $F\left(\lambda_{0}\right)$. Denote by $r_{i}$ the rank of $u_{0, i}$. If, for every $i=1, \ldots, I$, the vectors $u_{0, i}, \ldots, u_{r_{i}-1, i}$ form a Jordan chain consisting of an eigenvectors and associated vectors of $F(\lambda)$ at $\lambda_{0}$, then the system

$$
\left(u_{0, i}, u_{1, i}, \ldots, u_{r_{i}-1, i}\right)_{i=1, \ldots, I}
$$

is called a canonical system of Jordan chains corresponding to the characteristic value $\lambda_{0}$ of $F(\lambda)$.

Let $F(\lambda)$ be a holomorphic function in a punctured neighbourhood of $\lambda_{0}$ with values in $\mathcal{L}(B)$. Then we define the transposed family $F^{\prime}(\lambda)$ with values in $\mathcal{L}\left(B^{\prime}\right)$, where $B^{\prime}$ is the dual of $B$, by the equality $\left\langle F^{\prime} g, u\right\rangle=\langle g, F u\rangle$ for all $g \in B^{\prime}$ and $u \in B$.

The following result is proved by Gokhberg and Sigal [GS71] for meromorphic operator-valued functions as a consequence of their normal factorisation theorem. They refer to Keldysh [Kel51] for the case of polynomials with values in operators on a Hilbert space. 
Theorem 1.1. Let $\lambda_{0}$ be a characteristic value of the operator-valued function $F(\lambda)$, which is a normal point of $F(\lambda)$. Then there are biorthonormal canonical systems

$$
\begin{aligned}
& \left(u_{0, i}, u_{1, i}, \ldots, u_{r_{i}-1, i}\right)_{i=1, \ldots, I}, \\
& \left(g_{0, i}, g_{1, i}, \ldots, g_{r_{i}-1, i}\right)_{i=1, \ldots, I}
\end{aligned}
$$

of eigenvectors and associated vectors of $F(\lambda)$ and $F^{\prime}(\lambda)$ at $\lambda_{0}$, respectively, such that

$$
\text { p.p. } F^{-1}(\lambda)=\sum_{i=1}^{I} \sum_{j=-r_{i}}^{-1}\left(\lambda-\lambda_{0}\right)^{j} \sum_{k=0}^{r_{i}+j}\left\langle g_{k, i}, \cdot\right\rangle u_{r_{i}+j-k, i} .
$$

Here, the abbreviation p.p. indicates the principal part of the Laurent expansion around $\lambda_{0}$

\section{Definitions}

Let $\mathcal{D}$ be a bounded domain in $\mathbb{R}^{n}$ with boundary $\partial \mathcal{D}$ and let $\overline{\mathcal{D}}$ denote the closure of $\mathcal{D}$. We use the standard notation $x=\left(x_{1}, \ldots, x_{n}\right)$ for the coordinates in $\mathbb{R}^{n}$ and $D^{\alpha}=D_{1}^{\alpha_{1}} \ldots D_{n}^{\alpha_{1}}$ for the $\alpha$ th derivative, with $D_{j}=-\imath \partial / \partial x_{j}$ and $\alpha=\left(\alpha_{1}, \ldots, \alpha_{n}\right)$.

Assume that $\partial \mathcal{D}$ is a surface of the class $C^{2 m}$ everywhere away from the origin $x=0$ that we will denote by $O$. Moreover, in a neighbourhood of the point $O$ the domain $\mathcal{D}$ is assumed to coincide with a conical domain

$$
K=\left\{x \in \mathbb{R}^{n}: \frac{x}{|x|} \in \Omega\right\},
$$

where $\Omega$ is a domain on the unit sphere having a boundary of the class $C^{2 m}$.

Consider a differential operator in $\mathcal{D}$

$$
A(x, D)=\sum_{|\alpha| \leq 2 m} a_{\alpha}(x) D^{\alpha}
$$

where $a_{\alpha}(x)$ are bounded measurable functions in $\overline{\mathcal{D}}$. The higher order coefficients $a_{\alpha}(x)$ with $|\alpha|=2 m$ are required to be continuous in $\overline{\mathcal{D}} \backslash\{O\}$.

More precisely, the coefficients $a_{\alpha}(x)$ for $|\alpha|=2 m$ have the form

$$
a_{\alpha}(x)=a_{\alpha, 0}\left(\frac{x}{|x|}\right)+a_{\alpha, 1}(x)
$$

in a neighbourhood of the point $O$, where $\lim _{x \rightarrow 0} a_{\alpha, 1}(x)=0$.

We now introduce a system of boundary operators

$$
B_{j}(x, D)=\sum_{|\alpha| \leq m_{j}} b_{j, \alpha}(x) D^{\alpha}
$$

for $j=0, \ldots, m-1$, where $m_{j}<2 m$ and $b_{j, \alpha}(x)$ are functions of the class $C^{2 m-j}$ in $\overline{\mathcal{D}} \backslash\{O\}$. For $|\alpha|=m_{j}$ we require

$$
b_{j, \alpha}(x)=b_{j, \alpha, 0}\left(\frac{x}{|x|}\right)+b_{j, \alpha, 1}(x),
$$

where $\lim _{x \rightarrow 0} b_{j, \alpha, 1}(x)=0$.

In the sequel we assume that the operator $A$ is elliptic, i.e., $\sigma^{2 m}(A)(x, \xi) \neq 0$ for all $x \in \overline{\mathcal{D}} \backslash\{O\}$ and $\xi \in \mathbb{R}^{n} \backslash\{0\}$. If $n=2$ we also suppose that the condition of regular ellipticity is fulfilled. This latter means that for $x \in \mathcal{D}$ and any pair of linearly independent vectors $\xi, \eta$ the polynomial $\sigma^{2 m}(A)(x, \xi+z \eta)$ has exactly $m$ roots $z$ with positive imaginary part. It is known, cf. for instance [ADN59], that for $n>2$ this condition is always satisfied. It also holds if the coefficients of the principal symbol $\sigma^{2 m}(A)$ are real. 
Suppose that the Lopatinskii condition is fulfilled outside of the point $x=0$. The operators

$$
\begin{aligned}
A_{0} & =\sum_{|\alpha|=2 m} a_{\alpha, 0}(\omega) D^{\alpha}, \\
B_{j, 0} & =\sum_{|\alpha|=m_{j}} b_{j, \alpha, 0}(\omega) D^{\alpha},
\end{aligned}
$$

where $(r, \omega)$ is the spherical coordinate system with center at $O$, satisfy the Lopatinskii condition on $\partial K \backslash\{O\}$.

We shall consider complex-valued functions defined in $\mathcal{D}$. For $u \in C^{s}(\overline{\mathcal{D}})$, we introduce the norm

$$
\|u\|_{H^{s}(\mathcal{D})}=\left(\int_{\mathcal{D}} \sum_{|\alpha| \leq s}\left|D^{\alpha} u\right|^{2} d x\right)^{1 / 2} .
$$

The completion of the space $C^{s}(\overline{\mathcal{D}})$ with respect to this norm is the Banach space $H^{s}(\mathcal{D})$.

Given any $\gamma \in \mathbb{R}$, we define the space $H^{s, \gamma}(\mathcal{D})$ to consist of all functions $u$ such that

$$
\begin{aligned}
\|u\|_{H^{s, \gamma}(\mathcal{D})}^{2} & :=\int_{\mathcal{D}}|x|^{-2 \gamma} \sum_{|\alpha| \leq s}|x|^{2|\alpha|}\left|D^{\alpha} u\right|^{2} d x \\
& <\infty .
\end{aligned}
$$

Consider the boundary problem

$$
\begin{aligned}
A(x, D) u & =f \quad \text { in } \quad \mathcal{D}, \\
B_{j}(x, D) u & =0 \quad \text { on } \quad \partial \mathcal{D}
\end{aligned}
$$

for $j=0, \ldots, m-1$. The operator pencil

$$
\begin{aligned}
r^{-\imath \lambda} A_{0}\left(r^{\imath \lambda} u(\omega)\right), & \omega \in \Omega, \\
r^{-\imath \lambda} B_{j, 0}\left(r^{\imath \lambda} u(\omega)\right), & \omega \in \partial \Omega,
\end{aligned}
$$

is of crucial importance in the study of boundary value problems in domains with a conic point on the boundary. It is well known that the spectrum of the boundary value problem

$$
\begin{array}{r}
r^{-\imath \lambda} A_{0}\left(r^{\imath \lambda} u(\omega)\right)=0 \quad \text { for } \quad \omega \in \Omega, \\
r^{-\imath \lambda} B_{j, 0}\left(r^{\imath \lambda} u(\omega)\right)=0 \text { for } \quad \omega \in \partial \Omega
\end{array}
$$

is discrete.

The following theorem is proved in [Kon67].

Theorem 2.1. If there are no points of the spectrum of the problem (2.2) on the line $\Im \lambda=n / 2-\gamma$, then

$$
\|u\|_{H^{2 m, \gamma+2 m}(\mathcal{D})} \leq C\left(\|A u\|_{H^{0, \gamma}(\mathcal{D})}+\|u\|_{L^{2}(\mathcal{D})}\right)
$$

for all functions $u \in H^{2 m, \gamma+2 m}(\mathcal{D})$ satisfying the boundary condition $B_{j} u=0$ on $\partial \mathcal{D} \backslash\{O\}$ for each $j=0, \ldots m-1$.

\section{RAYS OF MINIMAL GROWTH}

Let us denote by $T$ the unbounded linear operator $L^{2}(\mathcal{D}) \rightarrow L^{2}(\mathcal{D})$ whose domain is

$$
\mathcal{D}_{T}=\left\{u \in H^{2 m, \gamma+2 m}(\mathcal{D}): B_{j} u=0 \text { on } \partial \mathcal{D} \backslash\{O\} \text { for } j=0, \ldots, m-1\right\}
$$

and that maps an element $u \in \mathcal{D}_{T}$ to $A u$.

Theorem 2.1 implies that $T$ is a closed linear operator $L^{2}(\mathcal{D}) \rightarrow L^{2}(\mathcal{D})$ and the dimensions of his kernel and cokernel are finite. If the spectrum of $T$ is not the whole complex plane then it is discrete. 
Definition 3.1. A ray $\arg \lambda=\theta$ in the complex plane $\lambda$ is called a ray of minimal growth for the resolvent $\mathcal{R}(\lambda)=(T-\lambda I)^{-1}: L^{2}(\mathcal{D}) \rightarrow L^{2}(\mathcal{D})$ of the operator $T$ if the resolvent exists for all $\lambda$ of sufficiently large modulus on this ray, and for all such $\lambda$ we have

$$
\|\mathcal{R}(\lambda)\|_{\mathcal{L}\left(L^{2}(\mathcal{D})\right)} \leq C|\lambda|^{-\delta}
$$

with some $\delta>0$ and a constant $C>0$.

Note that this definition is slightly different from the usual one where one assumes that $\delta=1$.

We will now indicate some conditions for a ray $\arg \lambda=\theta$ to be a ray of minimal growth for the resolvent of $T$.

Theorem 3.1. The spectrum of the operator $T$ is discrete and the ray $\arg \lambda=\theta$ is a ray of minimal growth for $\mathcal{R}(\lambda)$ if:

1) $\frac{A_{0}(x, \xi)}{\left|A_{0}(x, \xi)\right|} \neq e^{\imath \theta}$ for all $x \in \mathcal{D}$ and $\xi \in \mathbb{R}^{n} \backslash\{0\}$.

2) For $x \in \partial \mathcal{D} \backslash\{O\}$, let $\nu(x)$ be the normal vector for $\partial \mathcal{D}$ at $x$, and $\xi \in \mathbb{R}^{n} \backslash\{0\}$ be orthogonal to $\nu(x)$. If $z_{1}^{+}(\xi, \lambda), \ldots, z_{m}^{+}(\xi, \lambda)$ are the roots with positive imaginary parts of $A_{0}(x, \xi+z \nu(x))-\lambda$, where $\lambda$ is a complex number with $\arg \lambda=\theta$, then the polynomials $B_{j, 0}(x, \xi+z \nu(x)), j=0, \ldots, m-1$, are linearly independent modulo $\prod_{j=1}^{m}\left(z-z_{j}^{+}(\xi, \lambda)\right)$.

3) There is a $\gamma \in(-2 m, 0]$ with the property that the boundary value problem in the infinite cone $K$

$$
\begin{aligned}
A_{0}(\omega, D) u-e^{\imath \theta} u & =f \quad \text { for } \quad x \in K, \\
B_{j, 0}(\omega, D) u & =0 \quad \text { for } \quad x \in \partial K
\end{aligned}
$$

has a unique solution in $H^{2 m, \gamma+2 m}(K) \cap H^{0, \gamma}(K)$ for each $f \in H^{0, \gamma}(K)$, and

$$
\|u\|_{H^{2 m, \gamma+2 m}(K)}+\|u\|_{H^{0, \gamma}(K)} \leq C\|f\|_{H^{0, \gamma}(K)} .
$$

The conditions 1) and 2) were introduced by Agmon in [Agm62] where the completeness of the system of eigen and associated (root) vectors was proved for an elliptic boundary problem in a smooth domain. These conditions appear in the study of an elliptic boundary problem with a parameter. In the condition 2) the existence of $m$ roots with positive imaginary parts actually follows from the ellipticity of the operator $A$, which also implies that their number is less than or equal to $m$.

The condition 3 ) is difficult to check. It is met systematically in the study of boundary value problems in domains with conic points on the boundary, cf. for instance [MP79, NP94, Sch98]. One can show that the problem (3.1) is Fredholm, i.e., its kernel and cokernel have finite dimensions. The condition 3) says that these dimensions just amount to zero. This condition is actually equivalent to the following one:

$\left.3^{\prime}\right)$ For any $f(t, x)$ satisfying

$$
\int_{\mathbb{R}} \int_{K}|x|^{-2 \gamma}|f(t, x)|^{2} d t d x<\infty,
$$

there is a unique solution of the boundary value problem

$$
\begin{aligned}
e^{\imath \theta} D_{t}^{2 m} u-A_{0} u & =f \quad \text { in } \mathbb{R} \times K, \\
B_{j, 0} u & =0 \text { on } \mathbb{R} \times \partial K
\end{aligned}
$$

for $j=0, \ldots, m-1$, such that

$$
\int_{\mathbb{R}} \int_{K}|x|^{-2 \gamma-4 m}|u(t, x)|^{2} d t d x<\infty .
$$

There are some examples where this condition is fulfilled or is not. 


\section{Proof of Theorem 3.1}

We have to show that the estimate

$$
\|u\|_{L^{2}(\mathcal{D})} \leq \frac{C}{|\lambda|^{\delta}}\|(T-\lambda I) u\|_{L^{2}(\mathcal{D})}
$$

holds for any function $u \in \mathcal{D}_{T}$ and for all $\lambda$ with sufficiently large modulus on the ray $\arg \lambda=\theta$, where $\delta>0$.

Consider the operator

$$
L:=e^{\imath \theta} D_{t}^{2 m}-A
$$

The operator $L$ is elliptic of order $2 m$ in the closure of the cylindrical domain $\mathbb{R} \times \mathcal{D}$ of $\mathbb{R}^{n+1}$. One can check that 2 ) is equivalent to the condition that the operator $L$ and the system of boundary operators $B_{j}$ satisfy the Lopatinskii condition at each point of $\mathbb{R} \times(\partial \mathcal{D} \backslash\{O\})$.

Let $u(t, x) \in C^{2 m}(\mathbb{R} \times \overline{\mathcal{D}})$ be such that $u \equiv 0$ for all $|t| \geq 1$ and $B_{j} u=0$ on $\mathbb{R} \times(\partial \mathcal{D} \backslash\{O\})$ for each $j=0, \ldots, m-1$. Then the estimate

$$
\begin{aligned}
& \int_{\mathbb{R}} \int_{\mathcal{D}} r^{-2 \gamma}\left|D_{t}^{2 m} u\right|^{2} d t d x+\int_{\mathbb{R}} \int_{\mathcal{D}} r^{-2 \gamma-4 m} \sum_{|\alpha| \leq 2 m} r^{2|\alpha|}\left|D^{\alpha} u\right|^{2} d t d x \\
\leq & c\left(\int_{\mathbb{R}} \int_{\mathcal{D}} r^{-2 \gamma}|L u|^{2} d t d x+\int_{\mathbb{R}} \int_{\mathcal{D}}|u|^{2} d t d x\right)
\end{aligned}
$$

is true with a constant $c$ independent of $u$. Estimate (4.2) can be proved with the help of partition of unity and estimates of solutions to elliptic equations in $\mathbb{R}^{n}$, both in the half-space and in an unbounded dihedral angle. A detailed proof can be found in [Kon67].

Choose a function $\omega(t) \in C_{\text {comp }}^{\infty}(\mathbb{R})$ with the property that $\omega(t) \equiv 1$ for $|t|<1 / 2$ and $\omega(t)=0$ for $|t|>1$. Given a function $u(x) \in C^{2 m}(\overline{\mathcal{D}})$ satisfying the boundary conditions $B_{j} u=0$ on $\partial \mathcal{D} \backslash\{O\}$ for $j=0, \ldots, m-1$, we put

$$
v_{\sigma}(t, x)=\omega(t) e^{\imath \sigma t} u(x),
$$

where $\sigma$ is a real number.

For $\rho>0$, write $\mathcal{C}_{\rho}=(-\rho, \rho) \times \mathcal{D}$. Using inequality (4.2) we readily conclude that

$$
\int_{\mathcal{C}_{1}} r^{-2 \gamma-4 m} \sum_{|\alpha| \leq 2 m} r^{2|\alpha|}\left|D_{t, x}^{\alpha} v_{\sigma}\right|^{2} d t d x \leq c\left(\int_{\mathcal{C}_{1}} r^{-2 \gamma}\left|L v_{\sigma}\right|^{2} d t d x+\int_{\mathcal{C}_{1}}\left|v_{\sigma}\right|^{2} d t d x\right)
$$

for all $\sigma \in \mathbb{R}$, where the constant $c$ does not depend on $\sigma$. An easy computation shows that

$$
L v_{\sigma}=\omega(t) e^{\imath \sigma t}\left(\sigma^{2 m} e^{\imath \theta} u-A u\right)+S\left(e^{\imath \sigma t} u\right)
$$

where $S$ is a linear differential operator of order $2 m-1$ with bounded coefficients. Since $v_{\sigma} \equiv 0$ for $|t|>1$, (4.3) implies that

$$
\begin{aligned}
& \int_{\mathcal{C}_{1 / 2}} r^{-2 \gamma-4 m} \sum_{|\alpha| \leq 2 m} r^{2|\alpha|}\left|D_{t, x}^{\alpha}\left(e^{\imath \sigma t} u\right)\right|^{2} d t d x \\
\leq & C\left(\int_{\mathcal{D}} r^{-2 \gamma}\left|\sigma^{2 m} e^{\imath \theta} u-A u\right|^{2} d x+\int_{\mathcal{D}} r^{-2 \gamma} \sum_{|\alpha| \leq 2 m-1} \sigma^{2(2 m-1-|\alpha|)}\left|D^{\alpha} u\right|^{2} d x+\int_{\mathcal{D}}|u|^{2} d x\right),
\end{aligned}
$$


with $C$ a constant independent of $\sigma$ and $u$. Combining this inequality with an obvious estimate

$$
\begin{aligned}
& C^{\prime} \int_{\mathcal{C}_{1 / 2}} r^{-2 \gamma-4 m} \sum_{|\alpha| \leq 2 m} r^{2|\alpha|}\left|D_{t, x}^{\alpha}\left(e^{\imath \sigma t} u\right)\right|^{2} d t d x \\
& \geq \int_{\mathcal{D}} r^{-2 \gamma-4 m} \sum_{|\alpha|=2 m} \sum_{j+|\beta|=|\alpha|} r^{2|\alpha|} \sigma^{2 j}\left|D^{\beta} u\right|^{2} d x \\
& \geq c^{\prime}\left(\int_{\mathcal{D}} r^{-2 \gamma} \sum_{|\beta| \leq 2 m} \sigma^{2(2 m-|\beta|)}\left|D^{\beta} u\right|^{2} d x+\int_{\mathcal{D}} \sigma^{4 m+2 \gamma}|u|^{2} d x\right)
\end{aligned}
$$

we get

$$
\int_{\mathcal{D}} \sigma^{4 m+2 \gamma}|u|^{2} d x \leq \text { const } \int_{\mathcal{D}}\left|\sigma^{2 m} e^{\imath \theta} u-A u\right|^{2} d x
$$

for all $\sigma \in \mathbb{R}$ of sufficiently large modulus. This just amounts to saying that

$$
|\lambda|^{\frac{4 m+2 \gamma}{2 m}}\|u\|_{L^{2}(\mathcal{D})}^{2} \leq C\|(A-\lambda I) u\|_{L^{2}(\mathcal{D})}^{2}
$$

if $\arg \lambda=\theta$ and $|\lambda|$ is sufficiently large. Hence it follows that there are no points of the spectrum of the operator $A-\lambda I$ on the ray $\arg \lambda=\theta$ with modulus $|\lambda|$ large enough, and

$$
\left\|(T-\lambda I)^{-1}\right\|_{\mathcal{L}\left(L^{2}(\mathcal{D})\right)} \leq \sqrt{C}|\lambda|^{-1-\frac{\gamma}{2 m}} .
$$

Since we assume $-2 m<\gamma \leq 0$, the ray $\arg \lambda=\theta$ is a ray of minimal growth for the resolvent.

In order to finish the proof of Theorem 3.1 it remains to show that the map $T-\lambda I$ is a map onto the whole space $L^{2}(\mathcal{D})$. This proof is long and is based on usual methods of the theory of elliptic boundary problems with the help of partition of unity and construction of a parametrix.

\section{Generalised eigenelements}

Theorem 3.1 says that the spectrum of the operator $T$ is discrete. Let us fix a point $z$ outside of the spectrum of the operator $T$ and put $R=(T-z I)^{-1}$. We have

$$
\mathcal{R}\left(\frac{1}{\lambda-z}, R\right)=-(\lambda-z) I-(\lambda-z)^{2} \mathcal{R}(\lambda, T) .
$$

An element $\Phi \in L^{2}(\mathcal{D})$ different from zero is said to be a generalised eigenelement of the operator $R$ corresponding to an eigenvalue $\mu \in \mathbb{C}$, if $(R-\mu I)^{\iota} \Phi=0$ for some integer $\iota \geq 0$. The minimal $\iota$, for which this relation holds is then called the index of $\Phi$.

It is well known that the dimension of the space of generalised eigenelements of $R$ corresponding to an eigenvalue $\mu$ is finite. This dimension is called the multiplicity of $\mu$. Let us denote by $E(R)$ the closure in $L^{2}(\mathcal{D})$ of the linear span of all generalised eigenelements of the operator $R$.

The operator-valued function $\mathcal{R}(\lambda, R)$ is a meromorphic function of $\lambda \in \mathbb{C}$ with its poles at the points that are eigenvalues of the operator $R$. Let $f \in L^{2}(\mathcal{D})$. Consider the function $\mathcal{R}(\lambda, R) f$ which is analytic everywhere except of the point $\lambda=0$ and the points $\mu_{k}$ which can be its poles. If $\lambda=\mu_{k}$ is a pole of $\mathcal{R}(\lambda, R) f$ then in a sufficiently small neighbourhood of $\mu_{k}$ the function $\mathcal{R}(\lambda, R) f$ expands in a Laurent series

$$
\mathcal{R}(\lambda, R) f=\frac{\Phi_{\iota}}{\left(\lambda-\mu_{k}\right)^{\iota}}+\frac{\Phi_{\iota-1}}{\left(\lambda-\mu_{k}\right)^{\iota-1}}+\ldots+\frac{\Phi_{1}}{\lambda-\mu_{k}}+\sum_{j=0}^{\infty} f_{j}\left(\lambda-\mu_{k}\right)^{j},
$$

where $\iota \geq 1$ and $\Phi_{\iota} \neq 0$, the functions $\Phi_{j} \in L^{2}(\mathcal{D})$ are generalised eigenelements of $R$ of index $\iota-j+1$, and $f_{j} \in L^{2}(\mathcal{D})$ for $j \geq 0$. 
Similarly, a function $\Phi \in \mathcal{D}_{T}$ is said to be a generalised eigenelement of $T$ corresponding to an eigenvalue $\lambda$, if $(T-\lambda)^{\iota} \Phi=0$ for some $\iota \geq 1$. The minimal $\iota$, for which $(T-\lambda)^{\iota} \Phi=0$, is also called the index of $\Phi$.

It is clear that the function $\Phi$ is a generalised eigenelement of $T$ corresponding to $\lambda$ if and only if $\Phi$ is a generalised eigenelement of $\mathcal{R}(z, T)$, corresponding to the eigenvalue $1 /(\lambda-z)$. The closure in $L^{2}(\mathcal{D})$ of the linear span of all generalised eigenelements of the operator $T$ is denoted by $E(T)$. Out next objective is to show that $E(T)=L^{2}(\mathcal{D})$. But we first prove the following result about the growth of the resolvent.

Theorem 5.1. Suppose $R$ is a compact operator in $L^{2}(\mathcal{D})$ with the property that $R L^{2}(\mathcal{D}) \subset H^{2 m, \gamma+2 m}(\mathcal{D})$ for some $-2 m<\gamma<0$. Let $\left\{\mu_{\iota}\right\}$ be the sequence of nonzero eigenvalues of $R$ counted with their multiplicities, and $\mathcal{R}(\lambda, R)$ be the resolvent of $R$. Then

1) $\sum_{\iota}\left|\mu_{\iota}\right|^{\frac{n}{2 m+\gamma}+\varepsilon}<\infty$ for any $\varepsilon>0$.

2) There exists a sequence $\rho_{j} \rightarrow 0$, such that $\mathcal{R}(\lambda, R)$ is defined for $|\lambda|=\rho_{j}$ and satisfies

for $|\lambda|=\rho_{j}, j \in \mathbb{N}$, with any $\varepsilon>0$.

$$
\|\mathcal{R}(\lambda, R)\| \leq \exp \left(|\lambda|^{-\frac{n}{2 m+\gamma}-\varepsilon}\right)
$$

\section{Completeness of RoOT FunCtions}

To prove Theorem 5.1 we need some constructions from [Agm62]. Let $Q$ be a cube in $\mathbb{R}^{n}$,

$$
Q=\left\{x \in \mathbb{R}^{n}:\left|x_{j}\right|<\pi, j=1, \ldots, n\right\} .
$$

If $u \in L^{2}(Q)$ then

$$
u(x)=\sum_{k \in \mathbb{Z}^{n}} a_{k} e^{\imath\langle k, x\rangle} .
$$

For any $r>0$, let $H^{(r)}$ be the space of functions $u$ with finite norm

$$
\|u\|_{H^{(r)}}^{2}=\left|a_{0}\right|^{2}+\sum_{k \in \mathbb{Z}^{n} \backslash\{0\}}|k|^{2 r}\left|a_{k}\right|^{2} .
$$

Put

$$
\Lambda_{s} u(x)=\sum_{k \in \mathbb{Z}^{n}}\left(1+|k|^{2}\right)^{s / 2} a_{k} e^{\imath\langle k, x\rangle} .
$$

It is easy to verify that for $s>0$ the operator $\Lambda_{-s}$ is selfadjoint and compact in $L^{2}(Q)$. Its eigenvalues are $\left(1+|k|^{2}\right)^{-s / 2}$ and the corresponding eigenfunctions are $e^{\imath\langle k, x\rangle}$.

Let $z_{0}$ be a point not belonging to the spectrum of an operator $A$ in a Hilbert space $H$. Put $R=\left(A-z_{0} I\right)^{-1}$. We can certainly assume that $z_{0}=0$.

Obviously, $R^{*} R$ is a non-negative selfadjoint compact operator in $H$. The operator $S=\left(R^{*} R\right)^{1 / 2}$ is also non-negative, selfadjoint, and compact in $H$. Let $\mu_{j}(S)$ be the eigenvalues of $S$.

Definition 6.1. The operator $R$ is said to be of the class $C_{p}$, with $0<p<\infty$, provided

$$
\sum_{j}\left|\mu_{j}(S)\right|^{p}<\infty .
$$

Since

$$
\sum_{k \in \mathbb{Z}^{n}}\left(1+|k|^{2}\right)^{-p s / 2}<\infty
$$

if $p s>n$, the operator $\Lambda_{-s}$ belongs to $C_{p}$ for $p>n / s$. The following Lemmas are taken from [DS63]. 
Lemma 6.1. Assume that $R$ is a compact linear operator of the class $C_{p}$, with $0<p<\infty$, in a Hilbert space $H$. Then there exists a sequence $\rho_{j}$ satisfying $\rho_{j} \rightarrow 0$, such that

$$
\|\mathcal{R}(\lambda, R)\| \leq C \exp \left(c|\lambda|^{-p}\right)
$$

for $|\lambda|=\rho_{j}$.

Lemma 6.2. Let $R$ be a compact linear operator of the class $C_{p}$, with $0<p<\infty$, in a Hilbert space $H$, and $B$ be a bounded operator in $H$. Then the compositions $B R$ and $R B$ belong to $C_{p}$.

The following important result goes at least as far as [Agm62].

Theorem 6.3. Let $R$ be a compact operator in the Hilbert space $H^{(r)}$ for some $r \geq 0$ and $R H^{(r)} \subset H^{(r+s)}$ for some number $s>0$. Then $R \in C_{n / s+\varepsilon}$ for any $\varepsilon>0$ and

$$
\|C(\lambda) \mathcal{R}(\lambda, R)\| \leq \exp \left(c|\lambda|^{-n / s-\varepsilon}\right)
$$

whenever $\varepsilon>0$ and $|\lambda| \leq \Delta$ with $\Delta>0$ depending on $\varepsilon$, where

$$
C(\lambda)=\prod_{j}\left(1-\frac{\lambda_{j}}{\lambda}\right) \exp \left(\frac{\lambda_{j}}{\lambda}+\ldots+\frac{1}{N}\left(\frac{\lambda_{j}}{\lambda}\right)^{N}\right)
$$

and $N$ is the largest integer $\leq n / s$. The function $C(\lambda)$ is an entire function of $1 / \lambda$ vanishing at the points $\lambda_{j}$ only.

The condition $R H^{(r)} \subset H^{(r+s)}$ for some number $s>0$ can be replaced by requiring $R$ to be in the class $C_{p}$ for certain $p>0$. In this way one readily obtains a very useful consequence of Theorem 6.3, cf. [DS63]. It is also of independent interest.

Corollary 6.4. Assume that $R$ is a compact operator in a Hilbert space $H^{(r)}$ belonging to the class $C_{p}$, where $0<p<\infty$. Let $\lambda_{j}$ be the sequence of non-zero eigenvalues of $R$ counted with their multiplicities. Then there exists a sequence $\rho_{j}$ converging to 0 , such that the resolvent $\mathcal{R}(\lambda, R)$ exists everywhere on $|\lambda|=\rho_{j}$ and it fulfills

$$
\|\mathcal{R}(\lambda, R)\| \leq \exp \left(c|\lambda|^{-p}\right)
$$

for $|\lambda|=\rho_{i}$.

We shall show that Theorem 5.1 can be deduced from Corollary 6.4.

\section{Proof of Theorem 5.1}

Suppose that $\mathcal{D}$ is situated in the cube $Q=\left\{x:\left|x_{j}\right|<\pi, j=1, \ldots, n\right\}$. As usual, we denote by $r_{\mathcal{D}}$ the restriction operator from $L^{2}(Q)$ to $L^{2}(\mathcal{D})$. Let us show that there exists an extension operator $e_{\mathcal{D}}$ which maps $H^{2 m, \gamma+2 m}(\mathcal{D})$ continuously to $H^{2 m+\gamma}(Q)$, where $H^{2 m+\gamma}(Q)$ is the space of $2 \pi$-periodic functions on $\mathbb{R}^{n}$ with the norm

$$
\|u\|_{H^{s}(Q)}^{2}=\left|a_{0}\right|^{2}+\sum_{\substack{k \in \mathbb{Z}^{n} \\ k \neq 0}}|k|^{2 s}\left|a_{k}\right|^{2} .
$$

Here,

$$
u(x)=\sum_{k \in \mathbb{Z}^{n}} a_{k} e^{\imath\langle k, x\rangle}
$$

is the expansion of $u$ in the Fourier series, and $s=2 m+\gamma$. 
ON THE ROOT FUNCTIONS OF GENERAL ELLIPTIC BOUNDARY VALUE PROBLEMS 11

Consider in the infinite cone $K$ the partitions of unity

$$
\begin{aligned}
1 & =\sum_{i=-\infty}^{+\infty} \phi_{i}(x), \\
1 & =\sum_{i=-\infty}^{i+\infty} \psi_{i}(x),
\end{aligned}
$$

where

1) $\left.\left.\quad \phi_{i} \in C^{\infty}(K), \quad 2\right) \quad \operatorname{supp} \phi_{i} \subset K_{2^{-i-1} a, 2^{-i+1} a}, \quad 3\right) \quad\left|D^{\alpha} \phi_{i}\right| \leq C 2^{i|\alpha|}$;

$\left.\left.\left.1^{\prime}\right) \quad \psi_{i} \in C^{\infty}(K), \quad 2^{\prime}\right) \quad \operatorname{supp} \psi_{i} \subset K_{2^{-i-2} a, 2^{-i+2} a}, \quad 3^{\prime}\right) \quad\left|D^{\alpha} \psi_{i}\right| \leq C 2^{i|\alpha|}$

and $\psi=1$ in $K_{2^{-i-1} a, 2^{-i+1} a}$, i.e., $\phi_{i} \psi_{i}=\phi_{i}$ holds for all $i \in \mathbb{Z}$. Here, we define $K_{a, b}=\{x \in K: a<|x|<b\}$ and $K_{a}=K_{0, a}$.

Let $u_{1}=\phi u$ and $u_{2}=(1-\phi) u$, where $\phi \in C^{\infty}\left(\mathbb{R}^{n}\right)$ satisfies $\phi=1$ in a neighbourhood of the point $x=0$ and $\phi=0$ for $|x| \geq a$.

Set $B_{a, b}=\left\{x \in \mathbb{R}^{n}: a<|x|<b\right\}$ and let $e_{0}$ be a linear bounded extension operator from $H^{s}\left(K_{a / 4,4 a}\right)$ to $H^{s}\left(B_{a / 4,4 a}\right)$. The operator $e_{0}$ induces an extension operator $e_{i}$ from $H^{s}\left(K_{2^{-i-2} a, 2^{-i+2} a}\right)$ to $H^{s}\left(B_{2^{-i-2} a, 2^{-i+2} a}\right)$. Such an operator can be defined as follows.

Let $u^{\prime}(x)=u\left(2^{-i} x\right)$ for $x \in K_{a / 4,4 a}$, and $u^{\prime \prime}(x)=\left(e_{0} u^{\prime}\right)(x)$. We set

$$
\left(e_{i} u\right)(x)=u^{\prime \prime}\left(2^{i} x\right)
$$

for $x \in B_{2^{-i-2} a, 2^{-i+2} a}$. It is easy to verify that

$$
\left\|e_{i} u\right\|_{H^{s}\left(B_{2^{-i-2} a, 2^{-i+2 a}}\right)}^{2} \leq C \sum_{|\alpha| \leq s} \int_{K_{2^{-i-2 a, 2^{-i+2 a}}}} 2^{2 i(s-|\alpha|)}\left|D^{\alpha} u\right|^{2} d x
$$

with $C>0$ a constant independent of $u$.

We are now in a position to construct an extension operator $u \mapsto e(u)$ from $K$ to $\mathbb{R}^{n}$. Put

$$
e(u)=\sum_{i=1}^{+\infty} \psi_{i} e_{i}\left(\phi_{i} u\right)
$$

thus obtaining a continuation of $u$ from $K_{a}$ to $B_{a}$. 
If $|\beta| \leq 2 m$ then we obviously get

$$
\begin{aligned}
& \int_{B_{a}}|x|^{-2 \gamma-4 m+2|\beta|}\left|D^{\beta} e(u)\right|^{2} d x \\
& \leq C \sum_{i=1}^{\infty} \sum_{\beta^{1}+\beta^{2}=\beta} \int_{B_{2^{-i-2} a, 2^{-i+2} a}}|x|^{-2 \gamma-4 m+2|\beta|}\left|D^{\beta^{1}} \psi_{i}\right|^{2}\left|D^{\beta^{2}} e_{i}\left(\phi_{i} u\right)\right|^{2} d x \\
& \leq C \sum_{i=1}^{\infty} \sum_{\beta^{1}+\beta^{2}=\beta} \int_{B_{2^{-i-2} a, 2^{-i+2} a}} 2^{-i\left(-2 \gamma-4 m+2|\beta|-2\left|\beta^{1}\right|\right)}\left|D^{\beta^{2}} e_{i}\left(\phi_{i} u\right)\right|^{2} d x \\
& \leq C \sum_{i=1}^{\infty} \sum_{\beta^{1}+\beta^{2}=\beta} \sum_{|\alpha| \leq\left|\beta^{2}\right|} \int_{K_{2^{-i-2} a, 2^{-i+2} a}} 2^{-i(-2 \gamma-4 m+2|\alpha|))}\left|D^{\alpha}\left(\phi_{i} u\right)\right|^{2} d x \\
& \leq C \sum_{i=1}^{\infty} \sum_{|\alpha| \leq|\beta|} \sum_{\alpha^{1}+\alpha^{2}=\alpha} \int_{K_{2^{-i-1} a, 2^{-i+1} a}} 2^{-i\left(-2 \gamma-4 m+2\left|\alpha^{2}\right|\right)}\left|D^{\alpha^{2}} u\right|^{2} d x \\
& \leq C \sum_{i=1}^{\infty} \sum_{|\alpha| \leq|\beta|} \sum_{\alpha^{1}+\alpha^{2}=\alpha} \int_{K_{2^{-i-1} a, 2^{-i+1} a}}|x|^{-2 \gamma-4 m+2\left|\alpha^{2}\right|}\left|D^{\alpha^{2}} u\right|^{2} d x \\
& \leq C \int_{K_{a}}|x|^{-2 \gamma-4 m} \sum_{\left|\alpha^{2}\right| \leq 2 m}|x|^{2\left|\alpha^{2}\right|}\left|D^{\alpha^{2}} u\right|^{2} d x \\
& \leq C\|u\|_{H^{2 m, \gamma+2 m}(K)}^{2},
\end{aligned}
$$

the constant $C$, depending on $m, \gamma, \beta$ and $m$, may be different in diverse applications.

The function $\tilde{u}$, which is equal to $u$ in $\mathcal{D}$ and $e(u)$ in the punctured ball $B_{a}$, belongs to $H^{2 m, \gamma+2 m}\left(\mathcal{D} \cup B_{a}\right)$. The domain $\mathcal{D} \cup B_{a}$ is Lipschitzian, and we can extend $\tilde{u}$ to the cube $Q=\left\{x \in \mathbb{R}^{n}:\left|x_{j}\right| \leq a, j=1, \ldots, n\right\}$ in such a way that the continuation $U$ vanishes in a neighbourhood of $\partial Q$ and belongs to $H^{2 m, \gamma+2 m}(Q)$. Moreover,

$$
\|U\|_{H^{2 m, \gamma+2 m}(Q)} \leq c\|u\|_{H^{2 m, \gamma+2 m}(\mathcal{D})}
$$

with $c$ a constant independent of $u$.

Put $U=0$ outside of $Q$. Let us check that actually $U$ belongs to $H^{s}\left(\mathbb{R}^{n}\right)$ with $s<\gamma+2 m$ and

$$
\|U\|_{H^{s}\left(\mathbb{R}^{n}\right)} \leq C\|u\|_{H^{2 m, \gamma+2 m}(\mathcal{D})} .
$$

To do this, choose a partition of unity on $\mathbb{R}^{n} \backslash\{0\}$,

$$
1=\sum_{i=-\infty}^{\infty} \phi_{i}(x)
$$

such that $\phi_{i} \in C_{\text {comp }}^{\infty}\left(\mathbb{R}^{n}\right)$ is supported in $B_{2^{-i-1}, 2^{-i+1}}$ and $\left|D^{\alpha} \phi_{i}\right| \leq C 2^{i|\alpha|}$ for all $i$. Define $u_{i}=\phi_{i} U$.

The interpolation inequality implies that

$$
\varepsilon^{2 s}\left\|u_{i}\right\|_{H^{s}\left(\mathbb{R}^{n}\right)}^{2} \leq C\left(\varepsilon^{4 m} \sum_{|\alpha|=2 m} \int_{\mathbb{R}^{n}}\left|D^{\alpha} u_{i}\right|^{2} d x+\int_{\mathbb{R}^{n}}\left|u_{i}\right|^{2} d x\right),
$$

or

$$
\begin{aligned}
& \varepsilon^{2 s-4 m-2 \gamma}\left\|u_{i}\right\|_{H^{s}\left(\mathbb{R}^{n}\right)}^{2} \\
\leq & C\left(\varepsilon^{-2 \gamma} \sum_{|\alpha|=2 m} \int_{B_{2^{-i-1,2}-i+1}}\left|D^{\alpha} u_{i}\right|^{2} d x+\varepsilon^{-4 m-2 \gamma} \int_{B_{2^{-i-1}, 2^{-i+1}}}\left|u_{i}\right|^{2} d x\right) .
\end{aligned}
$$


Set $\varepsilon=2^{-i}$. Then

$$
\leq C\left(\sum_{|\alpha|=2 m} \int_{B_{2^{-i-1,2}-i+1}}|x|^{-2 \gamma}\left|D^{\alpha} u_{i}\right|^{2} d x+\int_{B_{2^{-i-1}, 2^{-i+1}}}|x|^{-2 \gamma-4 m}\left|u_{i}\right|^{2} d x\right),
$$

where $\delta=2 \gamma+4 m-2 s$ is positive. Since

$$
\begin{aligned}
\|U\|_{H^{s}\left(\mathbb{R}^{n}\right)} & \leq \sum_{i=-N}^{\infty}\left\|u_{i}\right\|_{H^{s}\left(\mathbb{R}^{n}\right)} \\
& \leq\left(\sum_{i=-N}^{\infty} 2^{i \delta}\left\|u_{i}\right\|_{H^{s}\left(\mathbb{R}^{n}\right)}^{2}\right)^{1 / 2}\left(\sum_{i=-N}^{\infty} 2^{-i \delta}\right)^{1 / 2}
\end{aligned}
$$

it follows that

$$
\begin{aligned}
& \|U\|_{H^{s}\left(\mathbb{R}^{n}\right)}^{2} \\
\leq & C \sum_{i=-N}^{\infty}\left(\sum_{|\alpha|=2 m} \int_{B_{2^{-i-1}, 2^{-i+1}}}|x|^{-2 \gamma}\left|D^{\alpha} u_{i}\right|^{2} d x+\int_{B_{2^{-i-1,2}-i+1}}|x|^{-2 \gamma-4 m}\left|u_{i}\right|^{2} d x\right) \\
\leq & C \sum_{i=-N}^{\infty} \int_{B_{2^{-i-1}, 2^{-i+1}}}|x|^{-2 \gamma-4 m} \sum_{|\alpha| \leq 2 m}|x|^{2|\alpha|}\left|D^{\alpha} u\right|^{2} d x \\
\leq & C\|u\|_{H^{2 m, \gamma+2 m}\left(\mathbb{R}^{n}\right)}^{2}
\end{aligned}
$$

which is majorised by $\|u\|_{H^{2 m, \gamma+2 m}(\mathcal{D})}^{2}$.

Therefore, the operator $e$ extends functions from $H^{2 m, \gamma+2 m}(\mathcal{D})$ continuously to $H^{s}\left(\mathbb{R}^{n}\right)$, for $s<\gamma+2 m$.

A function $u$ from $H^{2 m, \gamma+2 m}(Q) \cap H^{s}(Q)$ vanishing in a neighbourhood of $\partial Q$ can be extended to all of $\mathbb{R}^{n}$ as a $2 \pi$-periodic function. The norm of $u$ in $H^{s}\left(\mathbb{R}^{n}\right)$ is equivalent to the norm (7.1), if we put $u(x)=0$ outside of $Q$.

Let $R$ be a bounded linear operator from $H^{0, \gamma}(\mathcal{D})$ to $H^{2 m, \gamma+2 m}(\mathcal{D})$. Using the operator $e$ constructed above we introduce an operator $R_{Q}$ which acts in $L^{2}(Q)$ by

$$
R_{Q} u=e\left(R r_{\mathcal{D}} u\right),
$$

where $r_{\mathcal{D}}$ is the restriction operator from $L^{2}(Q)$ to $L^{2}(\mathcal{D})$. It is evident that the operator $R_{Q}$ is a compact operator from $L^{2}(Q)$ to $H_{s}(Q)$. Let us show that a $\lambda \neq 0$ belongs to the spectrum of the operator $R$ if and only if it belongs to the spectrum of the operator $R_{Q}$. Moreover, the multiplicity of $\lambda$ as a spectrum point of $R$ is the same as that of $R_{Q}$.

We first observe that if $u \in H^{2 m, \gamma+2 m}(\mathcal{D})$ then $R_{Q} e(u)=e(R u)$. Therefore, for any polynomial $p(z)$, we get

$$
p\left(R_{Q}\right) e(u)=e(p(R) u) .
$$

Furthermore, if $U \in H^{2 m, \gamma+2 m}(Q)$, then

$$
p(R) r_{\mathcal{D}} U=r_{\mathcal{D}} p\left(R_{Q}\right) U .
$$

Let $\lambda \neq 0$ be an eigenvalue of the operator $R$ and $\Phi(x) \in L^{2}(\mathcal{D})$ be an associated function of index $\iota \geq 1$, i.e., $(R-\lambda I)^{\iota} \Phi=0$ while $(R-\lambda I)^{\iota-1} \Phi \neq 0$. If $\iota=1$ then $\Phi$ is simply an eigenfunction.

Consider the polynomial

$$
p_{\iota-1}(z, \lambda)=\sum_{j=0}^{\iota-1}(z-\lambda)^{\iota-1-j}(-\lambda)^{j} .
$$


It is clear that

$$
\begin{aligned}
-(-\lambda)^{\iota} \Phi & =\left((R-\lambda I)^{\iota}-(-\lambda)^{\iota} I\right) \Phi \\
& =R p_{\iota-1}(R, \lambda) \Phi .
\end{aligned}
$$

Since $R$ is bounded as an operator acting from $L^{2}(\mathcal{D})$ to $H^{2 m, \gamma+2 m}(\mathcal{D})$, we see that $\Phi \in H^{2 m, \gamma+2 m}(\mathcal{D})$.

Let us now verify that if $\lambda \neq 0$ is a regular point of the operator $R$, then

$$
\begin{aligned}
\|\mathcal{R}(\lambda, R)\|_{\mathcal{L}\left(H^{0, \gamma}(\mathcal{D}), H^{2 m, \gamma+2 m}(\mathcal{D})\right)} & \leq c\left\|\mathcal{R}\left(\lambda, R_{Q}\right)\right\|_{\mathcal{L}\left(H^{0, \gamma}(Q), H^{2 m, \gamma+2 m}(Q)\right)} \\
& \leq C \frac{1}{|\lambda|}\left(\|\mathcal{R}(\lambda, R)\|_{\mathcal{L}\left(H^{0, \gamma}(\mathcal{D}), H^{2 m, \gamma+2 m}(\mathcal{D})\right)}+1\right)
\end{aligned}
$$

with $C$ a constant independent of $\lambda$.

It is easy to check that

$$
\mathcal{R}(\lambda, R) r_{\mathcal{D}} U=r_{\mathcal{D}} \mathcal{R}\left(\lambda, R_{Q}\right) U
$$

for all $U \in H^{0, \gamma}(Q)$, where $\mathcal{R}(\lambda, R)$ and $\mathcal{R}\left(\lambda, R_{Q}\right)$ are thought of as operators $H^{0, \gamma}(\mathcal{D}) \rightarrow H^{2 m, \gamma+2 m}(\mathcal{D})$ and $H^{0, \gamma}(Q) \rightarrow H^{2 m, \gamma+2 m}(Q)$, respectively. Indeed, we obtain

$$
\begin{aligned}
\mathcal{R}(\lambda, R) r_{\mathcal{D}} U & =\mathcal{R}(\lambda, R) r_{\mathcal{D}}\left(R_{Q}-\lambda I\right) \mathcal{R}\left(\lambda, R_{Q}\right) U \\
& =\mathcal{R}(\lambda, R)(R-\lambda I) r_{\mathcal{D}} \mathcal{R}\left(\lambda, R_{Q}\right) U \\
& =r_{\mathcal{D}} \mathcal{R}\left(\lambda, R_{Q}\right) U
\end{aligned}
$$

as desired.

Assuming $u \in H^{2 m, \gamma+2 m}(\mathcal{D})$ and substituting $U=e(u)$ into (7.4), we readily obtain

$$
\mathcal{R}(\lambda, R) u=r_{\mathcal{D}} \mathcal{R}\left(\lambda, R_{Q}\right) e(u)
$$

This relation implies $\|\mathcal{R}(\lambda, R)\| \leq C\left\|R\left(\lambda, R_{Q}\right)\right\|$ with $C$ a constant independent of $\lambda$.

Inversely if $U \in H^{0, \gamma}(Q)$, then by the definition of the operator $R_{Q}$ and (7.2) we easily get

$$
\begin{aligned}
\lambda \mathcal{R}\left(\lambda, R_{Q}\right) U & =R_{Q} \mathcal{R}\left(\lambda, R_{Q}\right) U-U \\
& =e R r_{\mathcal{D}} \mathcal{R}\left(\lambda, R_{Q}\right) U-U \\
& =e R \mathcal{R}(\lambda, R) r_{\mathcal{D}} U-U
\end{aligned}
$$

whence

$$
\left\|\mathcal{R}\left(\lambda, R_{Q}\right)\right\|_{\mathcal{L}\left(H^{0, \gamma}(Q), H^{2 m, \gamma+2 m}(Q)\right)} \leq \frac{C}{|\lambda|}\left(\|\mathcal{R}(\lambda, R)\|_{\mathcal{L}\left(H^{0, \gamma}(\mathcal{D}), H^{2 m, \gamma+2 m}(\mathcal{D})\right)}+1\right),
$$

where $C$ does not depend on $\lambda$. This proves the inequalities (7.3).

The operator $R=e \mathcal{R}(\lambda, T) r_{\mathcal{D}}$ mapping $H^{s}(Q)$ to $H^{s+\gamma+2 m-\varepsilon}(Q)$ is continuous. Its spectrum coincides with the spectrum of the operator $\mathcal{R}(\lambda, T)$. Moreover, the operator $\mathcal{R}(\lambda, T)=r_{\mathcal{D}} R e$ satisfies the conditions of Theorem 6.3 and Corollary 6.4 with $r=\gamma+2 m-\varepsilon$. Theorem 5.1 now follows immediately from Theorem 6.3 and Corollary 6.4.

\section{Main Result}

Now we are in a position to state our key result, i.e., the theorem on the completeness of the system of root functions of an elliptic boundary problem in a domain with a conical point on its boundary. 
Theorem 8.1. Suppose there are rays $\arg \lambda=\theta_{j}, j=1, \ldots, N$, in the complex plane which satisfy the hypotheses of Theorem 3.1 and such that the angles between the pairs of neighbouring rays are less than $\pi(\gamma+2 m) / n$. Then the spectrum of the operator $T$ is discrete and the root functions form a complete system in $L^{2}(\mathcal{D})$.

Proof. Theorem 3.1 implies that the spectrum of the operator $T$ is discrete and every ray $\arg \lambda=\theta_{j}$ is a ray of minimal growth for the resolvent $\mathcal{R}(\lambda, T)$ acting in $L^{2}(\mathcal{D})$. This means, in particular, that

$$
\|\mathcal{R}(\lambda, T)\|_{\mathcal{L}\left(L^{2}(\mathcal{D})\right)}=O\left(|\lambda|^{-\delta}\right)
$$

if $|\lambda| \rightarrow \infty$, with some $\delta>0$.

Assume that there exists a function $g \in L^{2}(\mathcal{D})$ which is orthogonal to all eigen and associated functions of the operator $T$. Our objective is to show that $g=0$. This will imply that the system of root functions is complete.

Suppose that the point $\lambda=0$ is regular for the operator $T$. Let us consider the function

$$
F(\lambda)=\left(\mathcal{R}\left(\frac{1}{\lambda}, R\right) f, g\right),
$$

where $R=T^{-1}, f \in L^{2}(\mathcal{D})$ and $(\cdot, \cdot)$ stands for the scalar product in $L^{2}(\mathcal{D})$.

Since the resolvent of $T$ is a meromorphic function with poles at the points of the spectrum of $T$, the function $F$ is analytic for those $\lambda$ which are not eigenvalues of $T$. We shall use a familiar relation between the resolvents of the operators $T$ and $T^{-1}$, namely

$$
\mathcal{R}\left(\frac{1}{\lambda}, T^{-1}\right)=-\lambda I-\lambda^{2} \mathcal{R}(\lambda, T)
$$

Consider the expansion

$$
\mathcal{R}(\lambda, R) f=\frac{\Phi_{\iota}}{\left(\lambda-\lambda_{k}\right)^{\iota}}+\frac{\Phi_{\iota-1}}{\left(\lambda-\lambda_{k}\right)^{\iota-1}}+\ldots+\frac{\Phi_{1}}{\lambda-\mu_{k}}+\sum_{j=0}^{\infty} f_{j}\left(\lambda-\lambda_{k}\right)^{j},
$$

in a neighbourhood of the point $\lambda=\lambda_{k}$, where $\lambda_{k}$ is a pole of $\mathcal{R}(\lambda, R)$. Here $\iota \geq 1$ and $\Phi_{\iota} \neq 0$, the functions $\Phi_{j} \in L^{2}(\mathcal{D})$ form a chain of associated functions of $R$, and $f_{j} \in L^{2}(\mathcal{D})$ for $j \geq 0$.

This expansion implies that $\lambda_{k}$ is a regular point of $F(\lambda)$, for $g$ is orthogonal to all $\Phi_{j}$. Therefore, $F(\lambda)$ is an entire function.

The relations (8.1), (8.2) and (8.3) imply that

$$
|F(\lambda)| \leq C \exp \left(|\lambda|^{2-\delta}\right)
$$

for $|\lambda| \rightarrow \infty$, provided that $\arg \lambda=\theta_{j}$ for some $j=1, \ldots, N$. Furthermore, Theorem 5.1 implies that for any $\varepsilon>0$ there is a sequence $\rho_{j} \rightarrow \infty$, such that

$$
|F(\lambda)| \leq \exp \left(|\lambda|^{-\frac{n}{2 m+\gamma}-\varepsilon}\right)
$$

for all $\lambda \in \mathbb{C}$ satisfying $|\lambda|=\rho_{j}$.

Consider $F(\lambda)$ in the closed corner between the rays $\arg \lambda=\theta_{j}$ and $\arg \lambda=\theta_{j+1}$. Its angle is less than $\pi(\gamma+2 m) / n$. Since

$$
\mathcal{R}\left(\frac{1}{\lambda}, R\right)=-\lambda I-\lambda^{2} \mathcal{R}(\lambda, T)
$$

and each ray $\arg \lambda=\theta_{k}$ is a ray of minimal growth, we have inequality (8.4) on the sides of the corner and (8.5) on a sequence of arcs tending to infinity.

Choosing $\varepsilon>0$ in (8.5) sufficiently small and applying the Fragmen-Lindelöf theorem we conclude that $|F(\lambda)|=O\left(|\lambda|^{2-\delta}\right)$ as $|\lambda| \rightarrow \infty$ in the whole complex plane. Therefore, $F(\lambda)$ is an affine function, i.e., $F(\lambda)=c_{0}+c_{1} \lambda$. On the other hand, we have

$$
\mathcal{R}(1 / \lambda, R)=-\lambda I-\lambda^{2} R+\ldots,
$$


and therefore,

$$
F(\lambda)=-\lambda(f, g)-\lambda^{2}(R f, g)+\ldots
$$

Since $F(\lambda)$ is affine, we get $(R f, g)=0$ for all $f \in L^{2}(\mathcal{D})$. Since the range of the operator $R$ is dense in $L^{2}(\mathcal{D})$, we deduce that $g=0$. Thus, the system of root functions of the operator $T$ is complete in $L^{2}(\mathcal{D})$.

\section{Some Generalisations}

We have proved the completeness of the system of eigen and associated functions of $T$ in $L^{2}(\mathcal{D})$. This theorem implies immediately the completeness in $H^{0, \gamma}(\mathcal{D})$ with any $\gamma<0$.

Indeed, let $f \in H^{0, \gamma}(\mathcal{D})$ where $\gamma<0$. Given any $\varepsilon>0$, put $f_{\varepsilon}=0$ for $|x|<\varrho$ and $f_{\varepsilon}=f$ for $|x|>\varrho$, where $\varrho>0$ is small enough, such that $\left\|f-f_{\varepsilon}\right\|_{H^{0, \gamma}(\mathcal{D})} \leq \varepsilon$. Since $f_{\varepsilon} \in L^{2}(\mathcal{D})$ there exists a finite linear combination of root vectors $L(x)$, such that

$$
\left\|f_{\varepsilon}-L\right\|_{L^{2}(\mathcal{D})} \leq \varepsilon
$$

Then

$$
\left\|f_{\varepsilon}-L\right\|_{H^{0, \gamma}(\mathcal{D})} \leq\left(\sup _{x \in \mathcal{D}}|x|^{-\gamma}\right) \varepsilon
$$

and

$$
\|f-L\|_{H^{0, \gamma}(\mathcal{D})} \leq\left(1+\sup _{x \in \mathcal{D}}|x|^{-\gamma}\right) \varepsilon .
$$

Now we shall state some consequences of Corollary 6.4.

Corollary 9.1. Let the conditions of Corollary 6.4 be fulfilled. Then the system of root elements is dense in the space

$$
H_{B}^{2 m, \gamma+2 m}(\mathcal{D})=\left\{u \in H^{2 m, \gamma+2 m}(\mathcal{D}): B u=0 \text { on } \partial \mathcal{D}\right\}
$$

for any $\gamma \leq 0$.

Proof. Indeed, pick a $u \in H_{B}^{2 m, \gamma+2 m}(\mathcal{D})$. Then $A u \in H_{B}^{0, \gamma}(\mathcal{D})$. Therefore, for any $\varepsilon>0$ there is a linear combination of root elements $L(x)$, such that

$$
\|A u-L\|_{H_{B}^{0, \gamma}(\mathcal{D})} \leq \varepsilon
$$

We can assume without loss of generality that $\lambda=0$ is a regular point of the spectrum of the operator $T$. The function $u_{0}=T^{-1}(L)$ is also a linear combination of root elements. It follows from (9.1) that

$$
\left\|u-u_{0}\right\|_{H^{2 m, \gamma+2 m}(\mathcal{D})} \leq C \varepsilon,
$$

where $C$ does not depend on $u$ and $\varepsilon$. The inequality (9.2) means that the system of eigen and associated functions is dense in $H^{2 m, \gamma+2 m}(\mathcal{D})$, as desired.

Corollary 9.2. Let the conditions of Corollary 6.4 be fulfilled, $\gamma \leq \gamma^{\prime} \leq 0$ and there be no spectrum points of the problem (4.3) in the strip

$$
\gamma+2 m-n / 2 \leq \Im \lambda \leq \gamma^{\prime}+2 m-n / 2 .
$$

Then the system of root elements is dense in the space $H_{B}^{2 m, \gamma^{\prime}+2 m}(\mathcal{D})$.

Proof. Indeed, let $u \in H_{B}^{2 m, \gamma^{\prime}+2 m}(\mathcal{D})$. Then $A u \in H^{0, \gamma^{\prime}}(\mathcal{D})$. Therefore, for any $\varepsilon>0$ there exists a linear combination of root elements $L(x)$, such that

$$
\|A u-L\|_{H^{0, \gamma^{\prime}(\mathcal{D})}} \leq \varepsilon
$$

Suppose $\lambda=0$ is a regular point of the spectrum of the operator $T$, which we can assume without loss of generality. The regularity theorem for solutions of elliptic boundary value problem in domains with conical points on the boundary implies that $\lambda=0$ is a regular point of the spectrum of $A: H^{2 m, \gamma^{\prime}+2 m}(\mathcal{D}) \rightarrow H^{0, \gamma^{\prime}}(\mathcal{D})$. 
The function $u_{0}=T^{-1}(L)$ is also a linear combination of root elements. It follows from (9.3) that

$$
\left\|u-u_{0}\right\|_{H^{2 m, \gamma^{\prime}+2 m}(\mathcal{D})} \leq C \varepsilon,
$$

with $C$ a constant independent of $u$ and $\varepsilon$. The inequality (9.4) shows that the system of eigen and associated functions is dense in $H^{2 m, \gamma^{\prime}+2 m}(\mathcal{D})$.

Since

$$
H^{2 m, \gamma^{\prime}+2 m}(\mathcal{D}) \hookrightarrow H^{0, \gamma^{\prime}+2 m}(\mathcal{D})
$$

and the space $C_{\text {comp }}^{\infty}(\mathcal{D})$ is dense in any $H^{0, \gamma^{\prime}+2 m}(\mathcal{D})$, we conclude that the system of root functions is dense in $H^{0, \gamma}(\mathcal{D})$ for $\gamma \leq 2 m$.

The obtained results can be extended to the spaces $L^{p}(\mathcal{D})$, for $p \geq 1$. The details are much the same as those in [Agm62].

Example 9.1. Consider an elliptic operator of second order

$$
A u:=\sum_{i, j=1}^{n} a_{i, j}(x) u_{x_{i} x_{j}}+\sum_{i=1}^{n} a_{i}(x) u_{x_{i}}+a_{0}(x) u,
$$

where $a_{i, j}, a_{i}$ and $a_{0}$ are continuous real-valued functions. We give $A$ the domain consisting of all $C^{2}$-functions which satisfy the homogeneous Dirichlet conditions in a domain with a finite number of conical points on its boundary. In the case where there are no conical points and the coefficients are smooth, the completeness was proved in [Agm62]. In our case the completeness follows from Theorem 8.1. It is worth pointing out that we can not apply the methods using the quadratic form $(A u, u)_{L^{2}(\mathcal{D})}$ as in [Agr94a] and [Kru76], since the coefficients $a_{i, j}$ can be not differentiable.

\section{SECOND ORDER EQUATIONS}

In this section we study the completeness of the system of eigenfunctions and associated functions, i.e., root functions, of the Neumann problem with zero data for a second order elliptic operator in the divergent form

$$
A u:=\sum_{i, j=1}^{n} \frac{\partial}{\partial x_{i}}\left(a_{i j}(x) \frac{\partial u}{\partial x_{j}}\right)+\sum_{i=1}^{n} a_{i}(x) \frac{\partial u}{\partial x_{i}}+a_{0}(x) u
$$

in the space $H^{1}(\mathcal{D})$.

We essentially weaken the conditions on the smoothness of the boundary of $\mathcal{D}$ and the coefficients of $A$. Namely, $\partial \mathcal{D}$ is assumed to be a Lipschitz surface, and $a_{i j}, a_{i}$ and $a_{0}$ bounded measurable real-valued functions in $\mathcal{D}$. As usual, we require $a_{i j}=a_{j i}$ and the uniform ellipticity

$$
\sum_{i, j=1}^{n} a_{i j}(x) \xi_{i} \xi_{j} \geq m|\xi|^{2}
$$

for all $x \in \mathcal{D}$ and $\xi \in \mathbb{R}^{n}$, with $m>0$ a constant independent of $x$ and $\xi$.

The result we obtain here are new even in case $A$ is the Helmholtz operator with zero Neumann data.

Our basic assumption is that an estimate

$$
\int_{\mathcal{D}}\left(\sum_{i, j=1}^{n} a_{i j} \frac{\partial u}{\partial x_{i}} \frac{\partial u}{\partial x_{j}}-\sum_{i=1}^{n} a_{i} \frac{\partial u}{\partial x_{i}} u-a_{0} u^{2}\right) d x \geq c \int_{\mathcal{D}}\left(|\nabla u|^{2}+u^{2}\right) d x
$$

is fulfilled for all $u \in H^{1}(\mathcal{D})$, with $c$ a constant independent of $u$. This assumption is not restrictive.

In particular, choosing $u$ constant yields

$$
\int_{\mathcal{D}} a_{0} d x<0 .
$$


A function $u \in H^{1}(\mathcal{D})$ is called a generalised solution of the Neumann problem with zero data for equation

$$
A u=\sum_{i=1}^{n} \frac{\partial f}{\partial x_{i}}+f_{0}
$$

where $f_{1}, \ldots, f_{n}$ and $f_{0}$ belong to $L^{2}(\mathcal{D})$, if for any $v \in H^{1}(\mathcal{D})$

$$
\int_{\mathcal{D}}\left(\sum_{i, j=1}^{n} a_{i j} \frac{\partial u}{\partial x_{j}} \frac{\partial v}{\partial x_{i}}-\sum_{i=1}^{n} a_{i} \frac{\partial u}{\partial x_{i}} v-a_{0} u v\right) d x=\int_{\mathcal{D}}\left(\sum_{i=1}^{n} f_{i} \frac{\partial v}{\partial x_{i}}-f_{0} v\right) d x .
$$

Using familiar functional methods one proves that the Neumann problem for (10.2) is uniquely solvable.

Lemma 10.1. Let (10.1) hold. Then the homogeneous Neumann problem for (10.2) has a unique generalised solution $u \in H^{1}(\mathcal{D})$ for all $f_{i}, f_{0} \in L^{2}(\mathcal{D})$. Moreover, we have

$$
\|u\|_{H^{1}(\mathcal{D})} \leq C\left(\sum_{i=1}^{n}\left\|f_{i}\right\|_{L^{2}(\mathcal{D})}+\left\|f_{0}\right\|_{L^{2}(\mathcal{D})}\right)
$$

with $C$ a constant independent of $f_{i}$ and $f_{0}$.

The fact that the root functions of the homogeneous Neumann problem for the operator $A$ are dense in $L^{2}(\mathcal{D})$ is proved in [Kru76], [Agr94b].

Theorem 10.2. Under the above assumptions, the root functions of the homogeneous Neumann problem for the operator $A$ are dense in $H^{1}(\mathcal{D})$.

Proof. Suppose $u_{0} \in H^{1}(\mathcal{D})$. Pick an arbitrary $\varepsilon>0$ and a function $u_{\varepsilon} \in C^{1}(\mathcal{D})$, such that

$$
\left\|u_{0}-u_{\varepsilon}\right\|_{H^{1}(\mathcal{D})}<\varepsilon \text {. }
$$

Set

$$
\begin{aligned}
& l_{i}(u)=\sum_{j=1}^{n} a_{i j}(x) \frac{\partial u}{\partial x_{j}}, \\
& l_{0}(u)=\sum_{i=1}^{n} a_{i}(x) \frac{\partial u}{\partial x_{i}}+a_{0}(x) u,
\end{aligned}
$$

for $i=1, \ldots, n$, and denote by $f_{h}$ the average of $f$ with step $h>0$. There exists an $h$ with the property that

$$
\sum_{i=1}^{n}\left\|l_{i}\left(u_{\varepsilon}\right)_{h}-l_{i}\left(u_{\varepsilon}\right)\right\|_{L^{2}(\mathcal{D})}+\left\|l_{0}\left(u_{\varepsilon}\right)_{h}-l_{0}\left(u_{\varepsilon}\right)\right\|_{L^{2}(\mathcal{D})}<\varepsilon .
$$

Since the root functions of the homogeneous Neumann problem for $A$ are dense in $L^{2}(\mathcal{D})$, there is a root function $U \in H^{1}(\mathcal{D})$, such that

$$
\left\|\left(\sum_{i=1}^{n} \frac{\partial}{\partial x_{i}} l_{i}\left(u_{\varepsilon}\right)_{h}+l_{0}\left(u_{\varepsilon}\right)_{h}\right)-U\right\|_{L^{2}(\mathcal{D})}<\varepsilon .
$$

If $v \in H^{1}(\mathcal{D})$ is a solution of

$$
A v=\sum_{i=1}^{n} \frac{\partial}{\partial x_{i}} l_{i}\left(u_{\varepsilon}\right)_{h}+l_{0}\left(u_{\varepsilon}\right)_{h}
$$

and $u \in H^{1}(\mathcal{D})$ is a root function satisfying $A u=U$, then estimate (10.3) readily implies that

$$
\|v-u\|_{H^{1}(\mathcal{D})} \leq C \varepsilon,
$$

the constant $C$ being as in Lemma 10.1. Moreover, it follows from (10.3) that

$$
\left\|u_{\varepsilon}-v\right\|_{H^{1}(\mathcal{D})} \leq C \varepsilon
$$


Combining (10.4) and (10.5) we get

$$
\begin{aligned}
\left\|u_{0}-u\right\|_{H^{1}(\mathcal{D})} & \leq\left\|u_{0}-u_{\varepsilon}\right\|_{H^{1}(\mathcal{D})}+\left\|u_{\varepsilon}-v\right\|_{H^{1}(\mathcal{D})}+\|v-u\|_{H^{1}(\mathcal{D})} \\
& <(1+2 C) \varepsilon .
\end{aligned}
$$

This shows that the root functions of the homogeneous Neumann problem for $A$ are dense in $H^{1}(\mathcal{D})$, as desired.

\section{REFERENCES}

[ADN59] Agmon, S., Douglis, A., Nirenberg, L., Estimates near the boundary for solutions of elliptic partial differential equations satisfying general boundary conditions, Comm. Pure Appl. Math. 12 (1959), 623-727.

[Agm62] Agmon, S., On the eigenfunctions and on the eigenvalues of general elliptic boundary value problems, Comm. Pure Appl. Math. 15 (1962), 119-147.

[Agr94] Agranovich, M. S., Elliptic operators on closed manifolds, In: Partial Differential Equations, VI, Encyclopedia of Mathematical Sciences, vol. 63, Springer, Berlin et al., 1994, $1-125$.

[Agr97] Agranovich, M. S., Elliptic boundary problems, In: Partial Differential Equations, IX, Encyclopedia of Mathematical Sciences, vol. 79, Springer, Berlin et al., 1997, 1-144.

[Agr94a] Agranovich, M. S., On series with respect to root vectors of operators associated with forms having symmetric principal part, Funct. Anal. Appl. 28 (1994), no. 3, 151-167.

[Agr94b] Agranovich, M. S., Non-selfadjoint elliptic problems on non-smooth domains, Russ. J. Math. Phys. 2 (1994), no. 2, 139-148.

[ADF00] Agranovich, M. S., Denk, R., Feierman, M., Weakly smooth non-selfadjoint spectral problems for elliptic boundary value problems, In: Spectral Theory, Microlocal Analysis, Singular Manifolds, Birkhäuser, Basel, 2000, 138-199.

[Car36] Carleman, T., Über die Verteilung der Eigenwerte partieller Differentialgleichungen, Ber. der Sächs. Akad. Wiss. Leipzig, Mat.-Nat. Kl. 88 (1936), 119-132.

[Bro53] Browder, F. E., On the eigenfunctions and eigenvalues of the general elliptic differential operator, Proc. Nat. Acad. Sci. USA 39 (1953), 433-439.

[Bro59a] Browder, F. E., Estimates and existence theorems for elliptic boundary value problems, Proc. Nat. Acad. Sci. USA 45 (1959), 365-372.

[Bro53b] Browder, F. E., On the spectral theory of strongly elliptic differential operators, Proc. Nat. Acad. Sci. USA 45 (1959), 1423-1431.

[DS63] Dunford, N., Schwartz J. T., Linear Operators, II, Selfadjoint Operators in Hilbert Space, Intersci. Publ., New York, 1963.

[EKS01] Egorov, Yu., Kondratiev, V., Schulze, B.-W., Completeness of eigenfunctions of an elliptic operator on a manifold with conical points, Russ. J. Math. Phys. 8 (2001), no. 3, 267-274.

[GS71] Gokhberg, I. Ts., Sigal, E. I., An operator generalisation of the logarithmic residue theorem and the theorem of Rouché, Math. USSR Sbornik 13 (1971), 603-625.

[Kel51] Keldysh, M. V., On the characteristic values and characteristic functions of certain classes of non-selfadjoint equations, Dokl. AN SSSR 77 (1951), 11-14.

[Kon67] Kondratiev, V. A., Boundary value problems for elliptic equations in domains with conical or singular points, Trudy Mosk. Mat. Ob. 16 (1967), 209-292.

[Kru76] Krukovsky, N. M., Theorems on the m-fold completeness of the generalized eigen- and associated functions from $W_{2}^{1}$ of certain boundary value problems for elliptic equations and systems, Diff. Uravneniya 12 (1976), no. 10, 1842-1851.

[Lid62] Lidskii, V. B., On summability of series in principal vectors of non-selfadjoint operators, Trudy Mosk. Mat. Ob. 11 (1962), 3-35.

[Mar62] Markus, A. S., Expansions in root vectors of a slightly perturbed selfadjoint operator, Dokl. Akad. Nauk SSSR 142 (1962), no. 3, 538-541.

[Mat64] Matsaev, V. I., On a method of estimating resolvents of non-selfadjoint operators, Dokl. Akad. Nauk SSSR 154 (1964), no. 5, 1034-1037.

[MP79] Maz'ya, V. G., Plamenevskii, B. A., On the asymptotics of the fundamental solutions of elliptic boundary value problems in domains with conic points, Probl. Math. Anal. 7 (1979), 100-145.

[NP94] Nazarov, S. A., Plamenevskii, B. A., Elliptic Problems in Domains with Piecewise Smooth Boundaries, De-Gruyter Expositions in Mathematics, 13, Berlin et al., 1994.

[Sch59] Schechter, M., Remarks on elliptic boundary value problems, Comm. Pure Appl. Math. 12 (1959), 457-482.

[Sch98] Schulze, B.-W., Boundary Value Problems and Singular Pseudo-Differential Operators, J. Wiley, Chichester, 1998. 
(Nikolai Tarkhanov) Institut für Mathematik, Universität Potsdam, Postfach 601553 , 14415 Potsdam, Germany

E-mail address: tarkhanov@math.uni-potsdam.de 